\title{
The III-V Triple-Junction Solar Cell Characteristics and Optimization with a Fresnel Lens Concentrator
}

\author{
Yin Guo, ${ }^{1,2}$ Qibing Liang, ${ }^{1,2}$ Bifen Shu $\mathbb{D}^{1,2}$ Jing Wang, ${ }^{1,2}$ and Qingchuan Yang ${ }^{1,2}$ \\ ${ }^{1}$ Institute for Solar Energy Systems, Sun Yat-Sen University, Guangzhou, China \\ ${ }^{2}$ Guangdong Provincial Key Laboratory of Photovoltaic Technologies, Guangzhou, China \\ Correspondence should be addressed to Bifen Shu; shubifen@163.com
}

Received 5 January 2018; Revised 13 March 2018; Accepted 29 March 2018; Published 9 May 2018

Academic Editor: Mahmoud M. El-Nahass

Copyright ( 2018 Yin Guo et al. This is an open access article distributed under the Creative Commons Attribution License, which permits unrestricted use, distribution, and reproduction in any medium, provided the original work is properly cited.

\begin{abstract}
At present, the Fresnel lens are commonly used as the condenser in high-concentrating photovoltaic (HCPV) modules. It is ideally believed that the output power of a III-V triple-junction solar cell which is placed on the focal plane of a Fresnel lens is the largest, because the intensity of the sunlight on the focal plane is the largest. Actually, according to our work, the dispersion of sunlight through a Fresnel lens and the nonparallelism and divergence of the incident light will lead to changes in the spectrum and the homogeneity of illumination, and cause a drop of the solar cell output. In this paper, the influence of the dispersion and nonparallel incidence of the light on the output of a triple-junction solar cell at different positions near the focal plane were theoretically studied, combined with the light-tracing simulation method and triple-junction solar cell circuit network model. The results show that the III-V triple-junction solar cell has the highest output power in both sides of the focal plane positions. The output power can be increased by about $15 \%$ after being optimized. The simulation results were verified by the experiments.
\end{abstract}

\section{Introduction}

The triple-junction cell is currently one of the most common multijunction cells used in high-concentrating photovoltaic (HCPV) modules. It is composed of three $\mathrm{p}-\mathrm{n}$ junctions in a series. Each subcell in it absorbs different wavelengths light [1-4]. HCPV modules based on Fresnel lens have now become a research hotspot $[5,6]$. Work was carried out for Fresnel lens design $[7,8]$ focused on improving the illumination energy on the cell, by improving the efficiency of the optical lens. However, the output power of the solar cell is not only related to the illumination energy but also to the spectral distribution and the uniformity of the illumination. The three subcells in a typical triple-junction solar cell respond to three spectral bands: the short band, 300$700 \mathrm{~nm}$; the medium band, 700-900 nm; and the long band, 900-1700 nm [9]. Owing to the dispersion, and considering that the refractive indices of different spectral bands are different, the illumination distributions of the three spectral bands are also different and nonuniform on the focal plane of lens.

Steiner et al. [10] discovered that the output current of their new multijunction solar cell decreased slightly on the designed focal plane of the lens. However, no work was carried out for further research. In this paper, we used the method of ray-tracing simulation [11] and the solar cell circuit network model [12-18] to analyze the illumination distribution and the power performance of the solar cell at different positions of the optical axis. The illumination distribution of different spectral bands and the power output performance of triple-junction solar cells were obtained.

\section{Mathematical Modelling}

2.1. The Dispersion of Fresnel Lens. Because a lens has different refractive indices for various frequencies of light, the direction of transmission of a monochromatic light is different after sunlight enters the lens, so it disperses when 


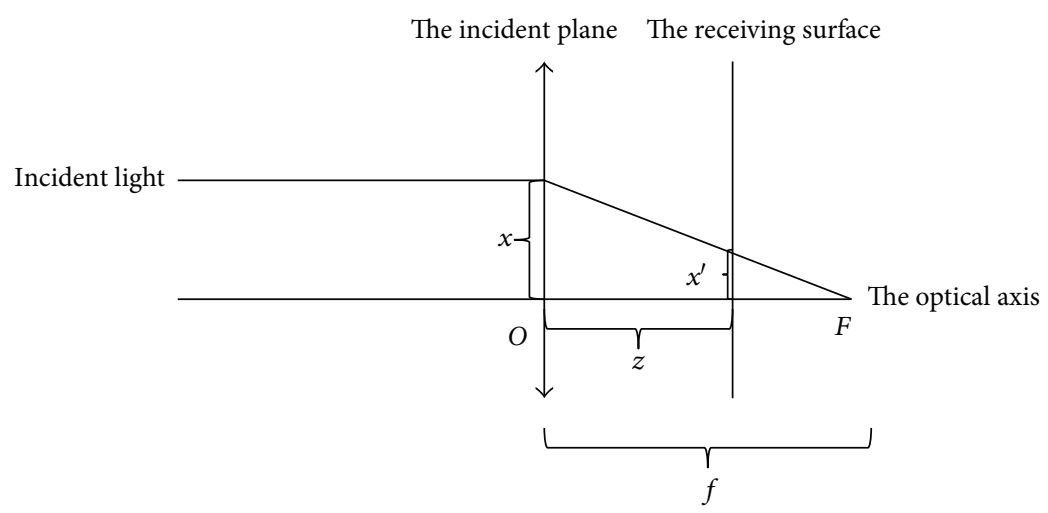

FIGURE 1: The light path of a normal incidence light passes through a Fresnel lens.

leaving the lens, and the colors are arranged in a certain order to form spectra. This is the dispersion phenomenon of the lens. Let us make a detailed analysis as shown below.

Figure 1 shows the normal incidence light passing through a Fresnel lens, where $x$ is the distance between the incident light and the optical axis, $x^{\prime}$ is the half width of the light spot on the receiving surface, $z$ is the distance between the receiving surface and Fresnel lens, $f$ is the focal length, and $F$ is the focal point.

By the geometric relation in Figure 1, there is

$$
\frac{x^{\prime}}{x}=\frac{f-z}{f} .
$$

The calculation formula above can be written as

$$
x^{\prime}=A x
$$

As you can see, given $f$ and $z$, a certain $x$ corresponding to a certain $x^{\prime}$, and $x$ and $x^{\prime}$ have the same distribution form. For a parallel light incident, with different positions $x_{1}$, $x_{2}, \ldots x_{n}$ of incident light, there is

$$
x_{n}^{\prime}=A x_{n} .
$$

Figure 2 shows the schematic diagram of a parallel incident light path. When the vertical incidence of light is uniformly distributed at the incident plane, the energy distribution of the convergent surface on the receiving surface is also uniform without considering energy loss caused by the refraction of light passing through the lens.

If $x$ is the maximum distance between the incident light and the optical axis, then $x^{\prime}$ is the maximum distance between the light spot of the receiving surface and the optical axis, so that the range of light for a given wavelength will be given at the given receiving surface. For different wavelengths of light, the focal length is different, so the light range on the receiving surface is also different.

Figure 3 is the light path diagram of a parallel polychromatic light. Here the definition of illumination width is half of the actual illumination range, represented by the subscript $m$, where $X_{m}$ is the width of incident light illumination, $x_{m \gamma}^{\prime}$ is the width of a certain wavelength of light on the receiving surface, and $f_{\gamma}$ is the focal length of a certain wavelength of light. There is

$$
x_{m \gamma}^{\prime}=\frac{\left|f_{\gamma}-z\right|}{f_{\gamma}} x_{m} .
$$

When position $Z$ is larger than $f$, it is still valid.

If the illumination distribution of incident light is uniform, it is also uniform for the light of a certain wavelength on the receiving surface, so that the illumination energy distribution of the receiving surface can be calculated. Set $w_{\gamma}$ as the unit wavelength light radiation power on the incident surface of the unit area and $w_{\gamma}^{\prime}$ as the unit wavelength light radiation power on the per receiving surface of the unit area. Because of the conservation of energy, there is

$$
w_{\gamma} d \gamma \cdot 4 x_{m}^{2}=w_{\gamma}^{\prime} d \gamma \cdot 4 x_{m \gamma}^{\prime 2}
$$

Therefore,

$$
w_{\gamma}^{\prime}=\frac{f_{\gamma}^{2}}{\left(f_{\gamma}-z\right)^{2}} w_{\gamma} .
$$

It can be seen that the illumination distribution of different wavelengths of light is generally different and the range of illumination is also different, so the illumination distribution on the receiving surface must be nonuniform. We can calculate the degree of nonuniformity of the illuminated distribution caused by the dispersion.

\subsection{Concentrating Illumination of Nonparallel and Divergent} Incident Light. For a monochromatic light at a certain wavelength, when the ray is not parallel to the optical axis, the light does not gather at the focal point of the lens but converges to a point on the plane passing through the focus and perpendicular to the axis; this plane is called the focal plane, as shown in Figure 4. When the incident light is divergent, the light in different directions converges at different points on the focal plane which improves the quality of the illumination energy near the focal point. The greater the divergence angle of the light source is, the more scattered the light energy will be. 


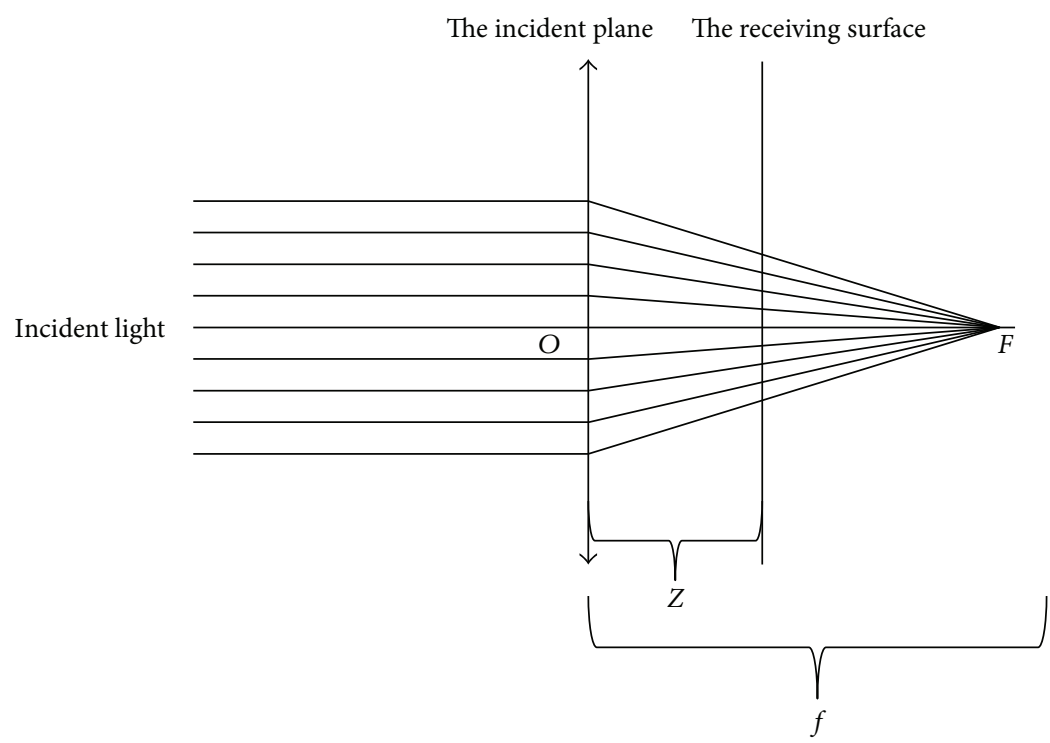

FIgURE 2: The schematic diagram of parallel incident light path.

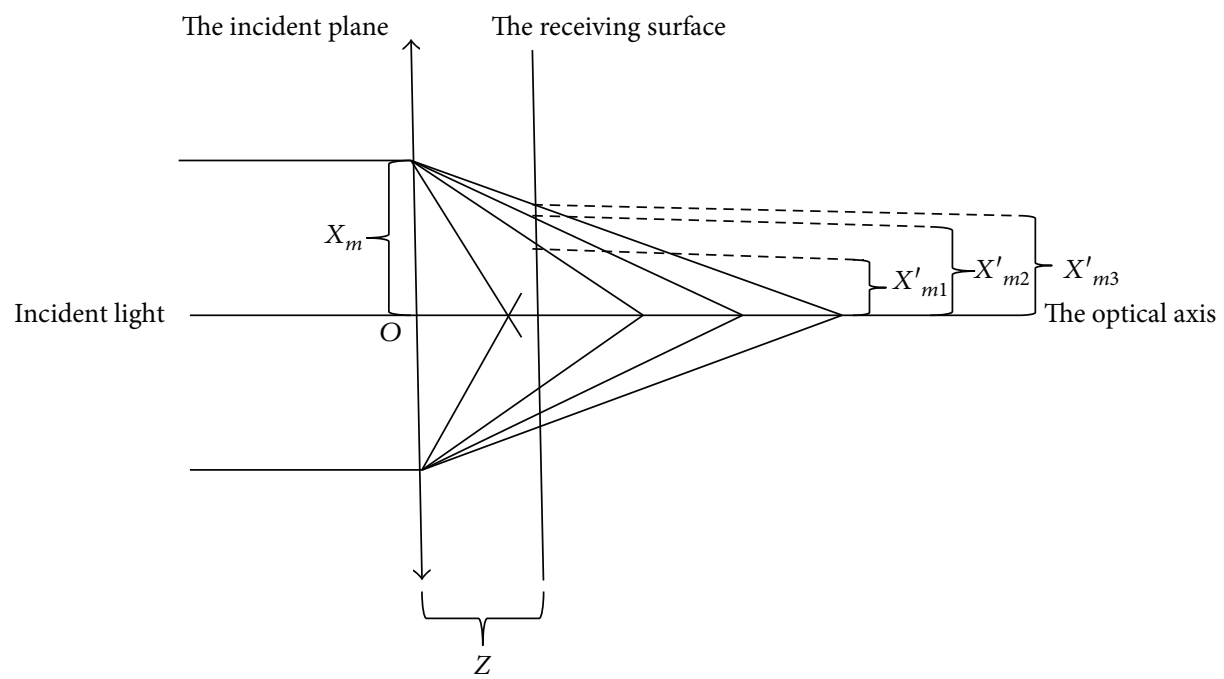

Figure 3: The light path diagram of parallel polychromatic light.

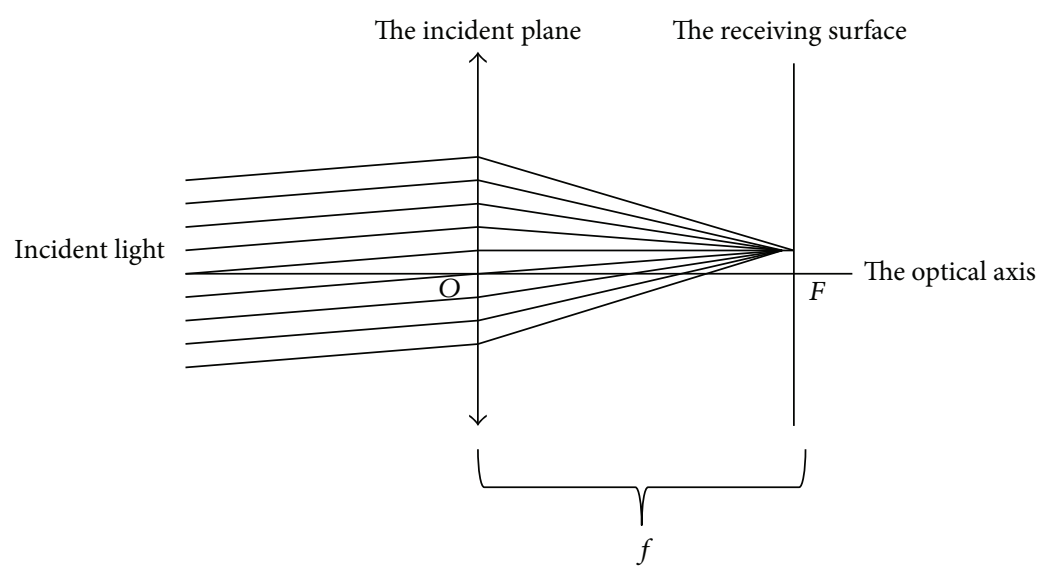

FIGURE 4: Schematic diagram of a nonparallel monochromatic incident light focusing through a lens. 


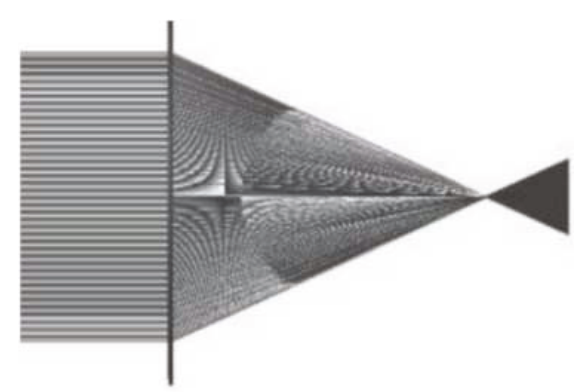

(a)

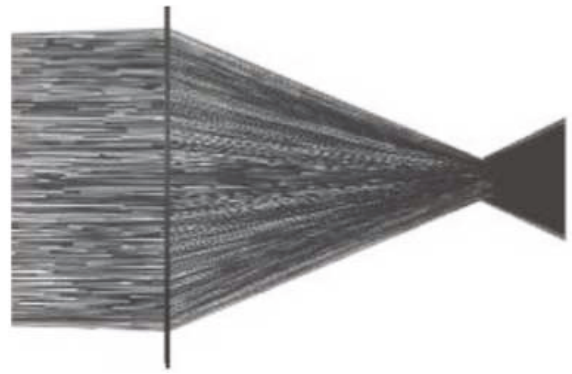

(c)

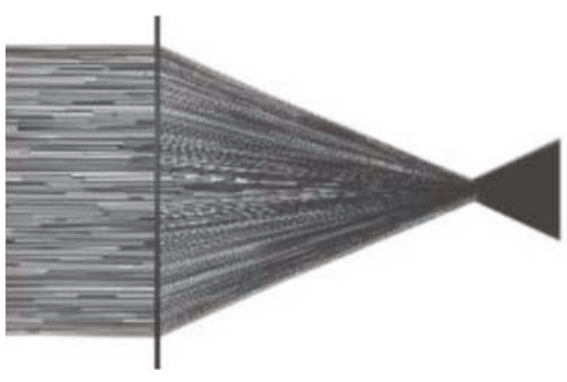

(b)

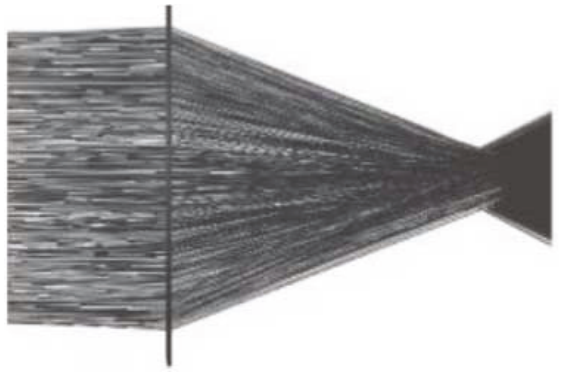

(d)

FIGURE 5: The diagram of the result of a $500 \mathrm{~nm}$ monochromatic incident light with different divergence angles concentrated by a lens. (a) Divergence angle at $0^{\circ}$. (b) Divergence angle at $1^{\circ}$. (c) Divergence angle at $2^{\circ}$. (d) Divergence angle at $3^{\circ}$.

Figures 5 and 6 are the results of our previous work [19]. Figure 5 shows the results of a $500 \mathrm{~nm}$ monochromatic incident light with different divergence angles concentrated by a lens. For parallel light, the light converges to the focal point, and if the solar cell is located at the focal point, the center of the cell will have great light intensity. When the light has a divergent angle, the light through the lens will not be concentrated entirely on the focal point. The larger the divergent angle is, the more scattered the spot near the focus will be, and the larger the spot is. The size of the spot is sensitive to the divergence angle of the light source, and the divergence angle, even at $1^{\circ}$, will have a very obvious effect on the spot size.

Figure 5 is the result of light for a single wavelength. The real situation is that the distribution of illumination on the solar cells is the result of the convergence of multiple wavelengths of light in the case of the spectral distribution of AM1.5d. Figure 6 is the distribution of the light intensity ratio on the surface of a solar cell at the focal point under the AM1.5d spectrum distribution, and the width of the cell is $10 \mathrm{~mm}$. The light intensity ratio here is defined as the ratio of the concentrated light intensity to the standard light intensity.

Figure 6 shows that the parallel incident light (divergence angle is $0^{\circ}$ ) is concentrated highly on the center of the cell. When the divergence angle increases, the light intensity ratio decreases, and the maximum light intensity ratio of the cell center decreases. This indicates that the divergence of light will obviously weaken the nonuniformity of the illumination on the solar cell surface. Because the nonuniformity of illumination has a negative impact on the performance of the cell, the divergence angle of the light source has a greater impact on the cell performance.
2.3. Circuit Network Model for Triple-Junction Solar Cells under Nonuniform Illumination. The equivalent circuit of the conventional triple-junction cell is shown in Figure 7(a). The triple-junction cell is composed of three $\mathrm{p}-\mathrm{n}$ junctions in the upper, middle, and lower parts, and each p-n junction is connected by the tunnel diode. The three junctions of the triple-junction cell are equivalent to three subcells, and each subcell is replaced by a dual-diode model. The equivalent circuit of a triple-junction cell can be used to solve the electrical characteristics of the cell under different concentration ratios and temperatures.

We know that triple-junction cells actually work under nonuniform illumination. In order to consider the influence of the heterogeneity of illumination on the cell performance, the cell can be divided into many smaller cells, with each smaller cell corresponding to different irradiation intensities at different locations. The basic idea of the circuit network model is to divide the cell into many smaller cells. The primary cell circuit is equivalent to a circuit formed by a parallel connection of many small cells. Each small cell has the electrical characteristics similar to the original cell. Each small cell is replaced by a dual diode model, respectively. The cross connection between smaller cells, the contact between the surface and the metal grid of the original cell, and the interconnection of the metal grid itself, are connected by resistances. The circuit network is shown in Figure 7(b) [19].

\section{Results and Analysis}

3.1. Concentrating Characteristics of Fresnel Lens. In order to study the photovoltaic characteristics of triple-junction cells under Fresnel lens concentrators, we first studied the concentrating characteristics of Fresnel lens for three bands of solar 


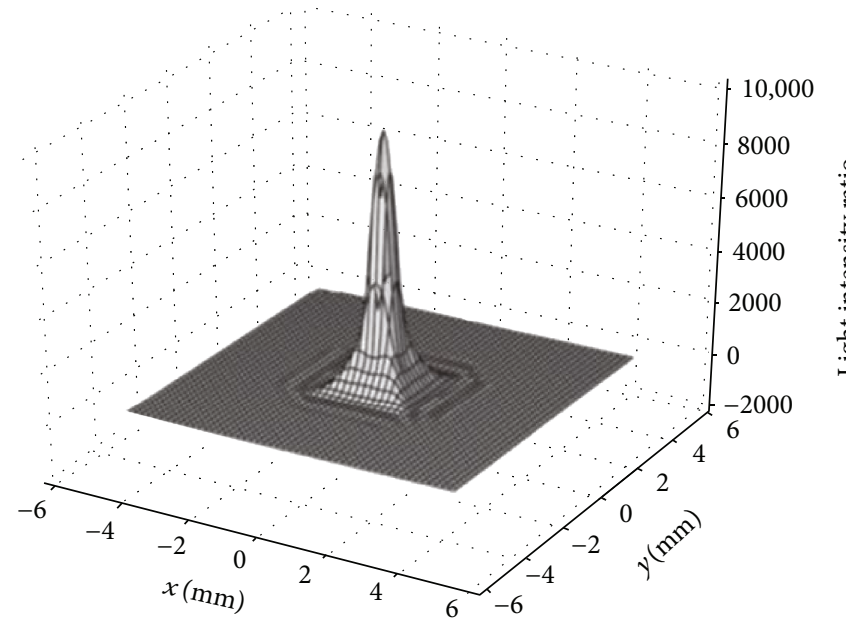

(a)

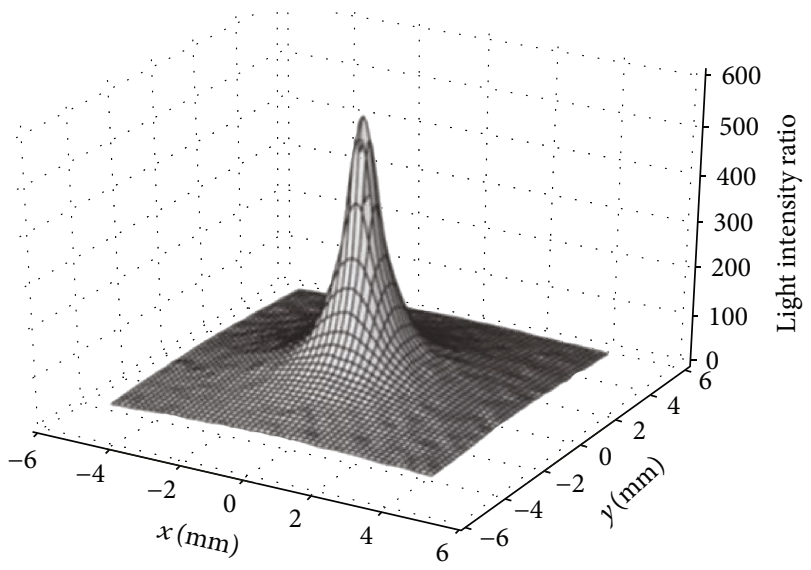

(c)

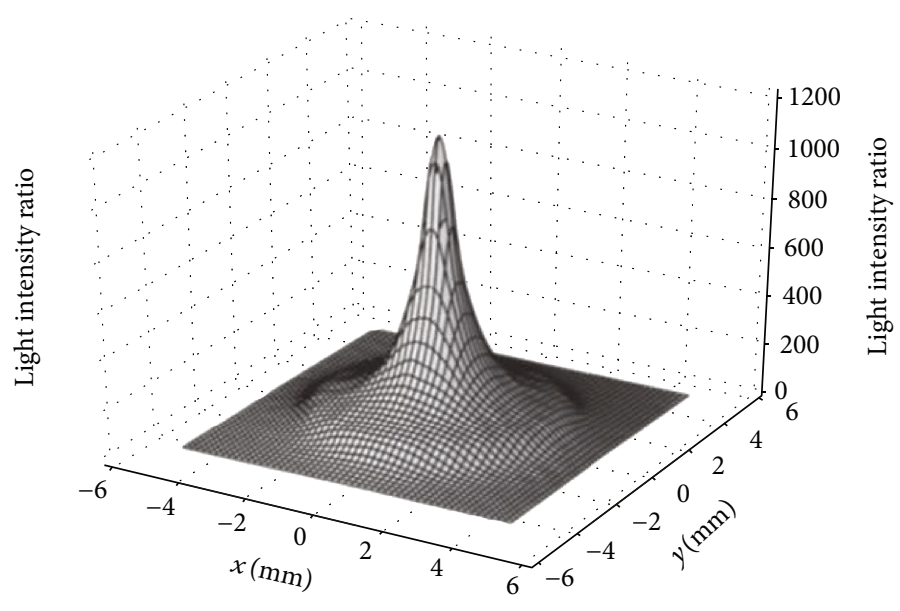

(b)

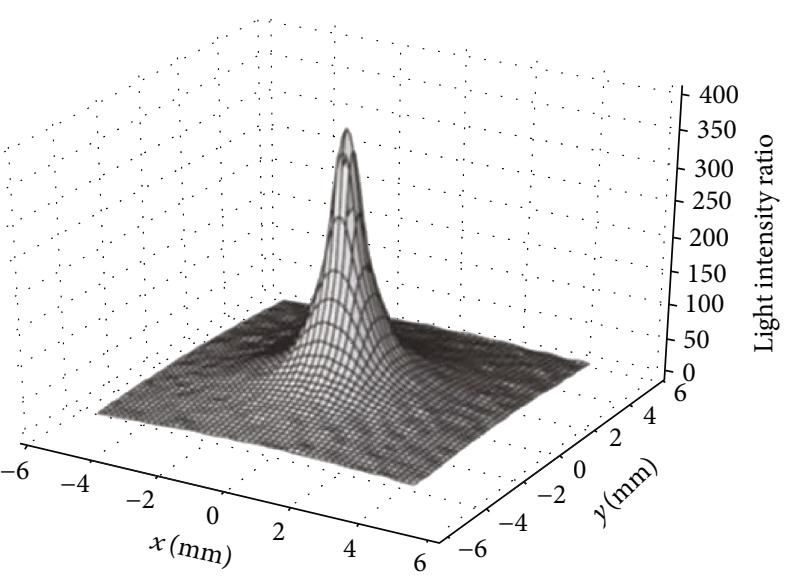

(d)

FIgure 6: The distribution of the light intensity ratio on the surface of a solar cell at the focal point under the AM1.5d spectrum distribution; the width of the cell is $10 \mathrm{~mm}$. (a) Divergence angle at $0^{\circ}$. (b) Divergence angle at $1^{\circ}$. (c) Divergence angle at $2^{\circ}$. (d) Divergence angle at $3^{\circ}$.

light (short: $300-700 \mathrm{~nm}$, medium: $700-900 \mathrm{~nm}$, and long: 900-1700). These three bands correspond to the three subcells of triple-junction cells.

Figure 8 shows the variation of the spot diameter of three bands with the position of the optical axis under a Fresnel lens for parallel incident light. For $Z$ value, $Z=0$ is the focal plane of the lens of full-band solar light. The negative value $Z$ is expressed above the focal plane, close to the Fresnel lens, and the positive value $Z$ is expressed below the focal plane and far away from the lens. The Fresnel lens material is PMMA.

It can be seen from Figure 8 that the diameter of the spot of the three bands below the Fresnel lens varies with the change of the optical axis position. For the shortwave band, there is a minimum spot diameter of $3.7 \mathrm{~mm}$ at $5.5 \mathrm{~mm}$ above the focal plane. The minimum spot diameter is the same as $0.8 \mathrm{~mm}$ of the middle and long wavelengths, but the location is slightly different. The middle band is $4.5 \mathrm{~mm}$ below the focal plane, and the long band is $5.5 \mathrm{~mm}$ below the focal plane. At the focal plane position of the lens, the diameter of the short-band light spot is $5.8 \mathrm{~mm}$, and the diameter of the middle-long-band light spot is about $2 \mathrm{~mm}$. The difference of the diameter of the light spot in different bands shows that the degree of nonuniformity of light in different bands is different for a certain size of the cell.

Figure 9 shows that the light spots vary with the installation position of the triple-junction cell in the experimental test. The size of the III-V triple-junction cell is $5 \mathrm{~mm}^{*} 5 \mathrm{~mm}$. The Fresnel lens is $100 \mathrm{~mm}^{*} 100 \mathrm{~mm}$ with a focal length of $110 \mathrm{~mm}$, and the material of the lens is PMMA. The outdoor experiment is carried out on the roof of our Institute in Guangzhou. The DNI is about $700-800 \mathrm{~W} / \mathrm{m}^{2}$, and the atmospheric temperature is about $25^{\circ} \mathrm{C}$.

From Figure 9 we can observe that when the distance between the triple-junction cell and the lens changes, the spot on the cell will change significantly. In the process of moving from $5 \mathrm{~mm}$ above the focal plane to $5 \mathrm{~mm}$ below the focal plane, no change can be observed to the overall size of the spot, almost covering the surface of the cell, while the spot color slowly changed from red and orange to blue and violet. We have known from Figure 8 that the refractive indices of different wave bands of sunlight are different, so the illumination distribution of different bands in the optical axis will be different. Figure 9 shows that when $Z=-5 \mathrm{~mm}$, the light spot 


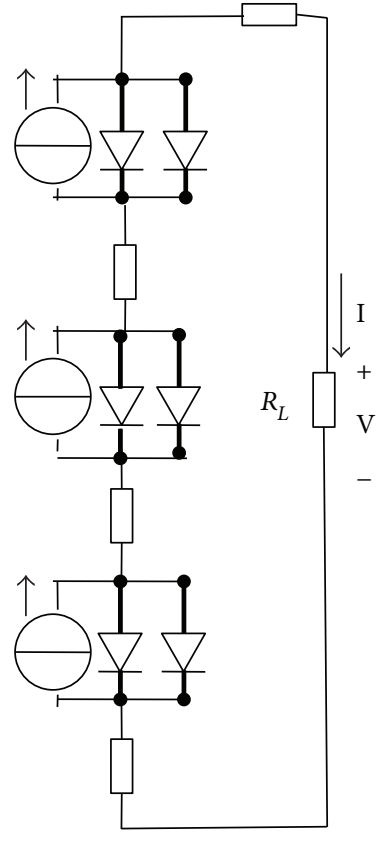

(a)

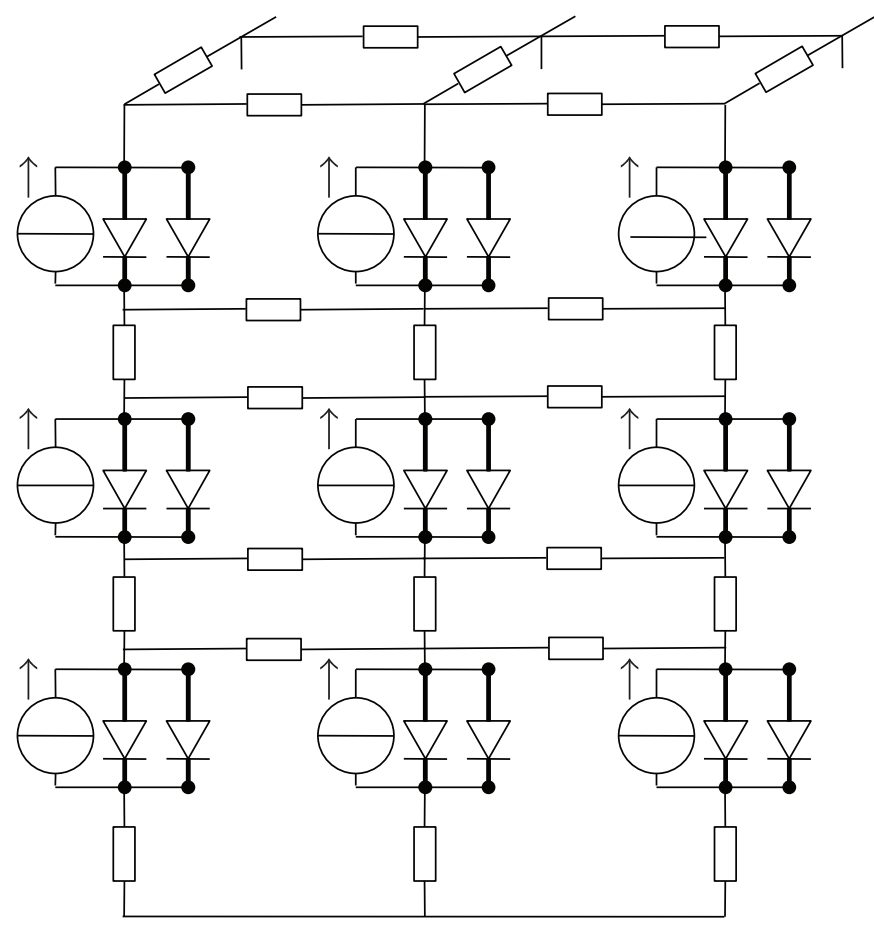

(b)

FIgURE 7: The circuit network model of triple-junction cells under nonuniform illumination.

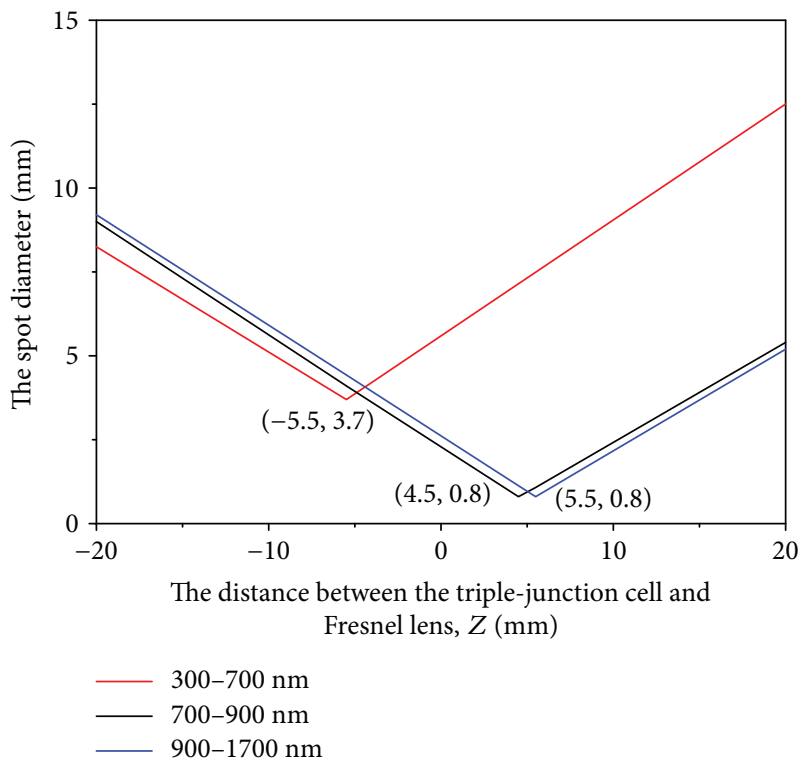

FIGURE 8: The variation of the spot diameter of three bands with the position of the optical axis under Fresnel lens.

of the short-wavelength band is small and the light spot of the long-wavelength band is larger, so the outer ring of the spot is observed to be orange red. When $Z=5 \mathrm{~mm}$, the short-wavelength band light spot is large and the longwavelength light spot is smaller, so the outer ring of the spot is blue purple. When $Z=0 \mathrm{~mm}$ (the focal plane), the three bands of light converge to be white light, and the light spot is relatively concentrated.
Because a triple-junction cell is composed of three subcells absorbing three different wave bands of light, the differences of illumination distribution on the triple-junction cell surface will inevitably affect the power output performance of triple-junction cells.

3.2. Triple-Junction Solar Cell Characteristics. Sunlight can be considered as parallel light. However, due to factors such as dust and the limitations of lens-processing technology, light will inevitably diverge after passing through a lens. So we simulated the photovoltaic characteristics of triple-junction cells under the illumination of light sources with different divergence angles.

Figure 10 is the change of the short circuit current, maximum-output power, fill factor, and efficiency of a $5 \mathrm{~mm}^{*} 5 \mathrm{~mm}$ triple-junction cell with the position of the optical axis when the divergence angles of a light source are $0^{\circ}$, $0.1^{\circ}, 0.2^{\circ}, 0.5^{\circ}, 1^{\circ}, 2^{\circ}$, and $4.4^{\circ}$ respectively.

Seen from Figure 10, at the focus of the lens (focal plane) position, when the incident light is more parallel (divergence angle less than 0.2 degrees), the short circuit current of the cell is greatly reduced. This is because the differences of the intensity of the short-, medium-, and long-light bands is large, and it leads to the current mismatch of the three subcells. Moreover, the illumination distribution is very nonuniform, so the fill factor (FF) is also very low, causing the efficiency and the output power of the cell to be very low. When the divergence degree is greater than $0.2^{\circ}$ (or equal to $0.2^{\circ}$ ) and less than $0.5^{\circ}$, the focal spot becomes larger, which is almost the same as the area of the cell. The nonuniform degree of illumination distribution on the cell decreases, so the short circuit current and fill factor are all high, which 


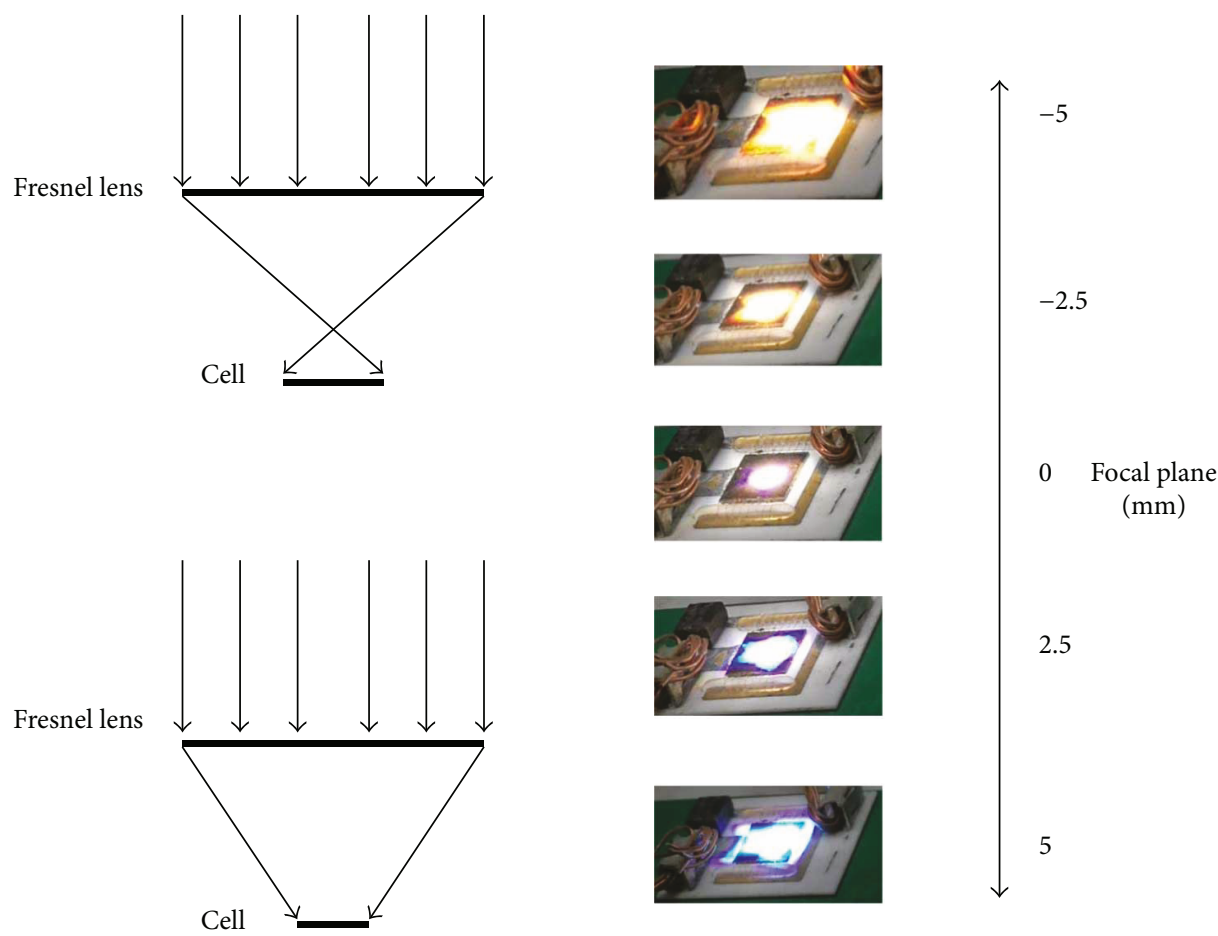

FIGURE 9: Light spots vary with the installation position of the triple-junction cell (along the direction of the optical axis).

makes the efficiency and output power larger. When the divergence is greater than 0.5 degrees, the spot is too scattered, and its area is larger than the area of the cell. Parts of the illumination were out of the cell. Although the illumination on the cell is more homogeneous, the energy decreases. So although the fill factor is larger, the short circuit current is too low, which makes the cell efficiency and output power smaller.

From the results of Figure 10, we also find that when the divergence degree is 0.2 degrees, the performance of the cell is the best if the cell is located at the upper and lower sides of the focal plane $(Z=-9 \mathrm{~mm}, Z=11 \mathrm{~mm})$. We know that, in practical application, sunlight can be considered as parallel light. However, due to factors such as dust and limitations of lens-processing technology, light will inevitably diverge after passing through the lens. Normally, it is considered at about 0.2 degrees. Therefore, we can determine the position of the upper and lower sides of the focal plane as the optimal position of the cell performance. The maximum-output power of the cell $(2.7 \mathrm{~W})$ at this optimal position is about $17 \%$ higher than that at the focal plane $(2.3 \mathrm{~W})$.

In order to verify the results of the numerical simulation, we use the existing experimental platform to carry out the experiment. The experimental platform and device are shown in Figure 11. The test cell is a triple-junction cell with an area of $5 \mathrm{~mm}^{*} 5 \mathrm{~mm}$.

We tested the short circuit current of the triple-junction solar cell in the different optical axis positions many times. We found that the short circuit current in different axial positions presents regular distribution.

Figure 12 shows the influence of the optical axis position on the short circuit current of the triple-junction cell. It uses repeated testing data 3 times, and tests 20 experimental points from $5 \mathrm{~mm}$ above the focal plane to $5 \mathrm{~mm}$ below the focal plane, with a $0.5 \mathrm{~mm}$ interval.

Neglecting the influence of some outdoor measurement errors, we can see from the diagram that the short circuit current of the triple-junction cell has a minimum value at the focal plane, and there is a maximum value at the both sides of the focal plane. Compared with the focus position, the short circuit current at both sides of the focus increases by about $10 \%$.

Figure 13 shows the change in the maximum-output power of the triple-junction cell with the position of the optical axis. We can see from the diagram above, the maximumoutput power also has a minimum value at the focal plane, and there is a maximum value at both sides of the focal plane. Compared with the focus position, the maximum-output power at both sides of the focus increases by about $15 \%$.

Figure 14 shows the changes in the fill factor of the triplejunction cell with the position of the optical axis. It can be seen that the change regulation of the fill factor is similar to that of the maximum-output power. Compared with the focus position, the fill factor at both sides of the focus also increases by about $15 \%$. It can be found from the results above that the experimental results are in agreement with the simulated results.

\section{Conclusion}

In this paper, we first establish theoretical models to simulate the concentrating characteristics of Fresnel lens, considering the effects of the lens dispersion and the nonparallel incidence of light. It is found that for different bands of light, 

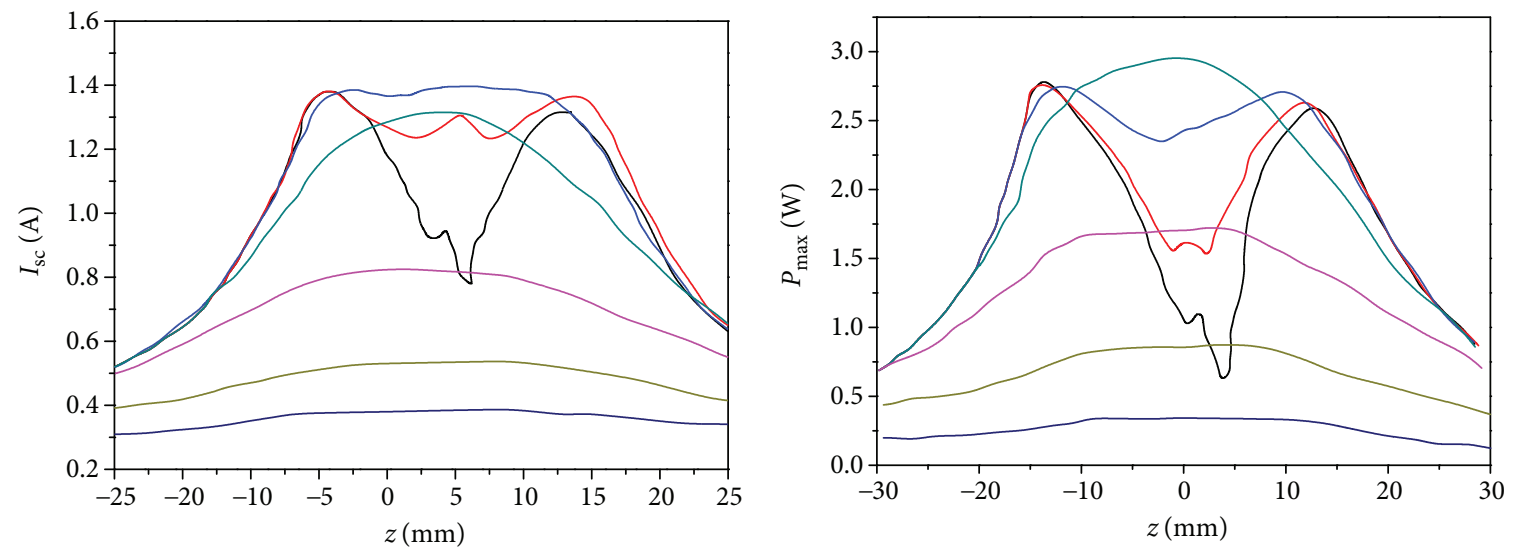

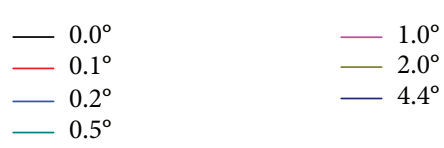

(a)
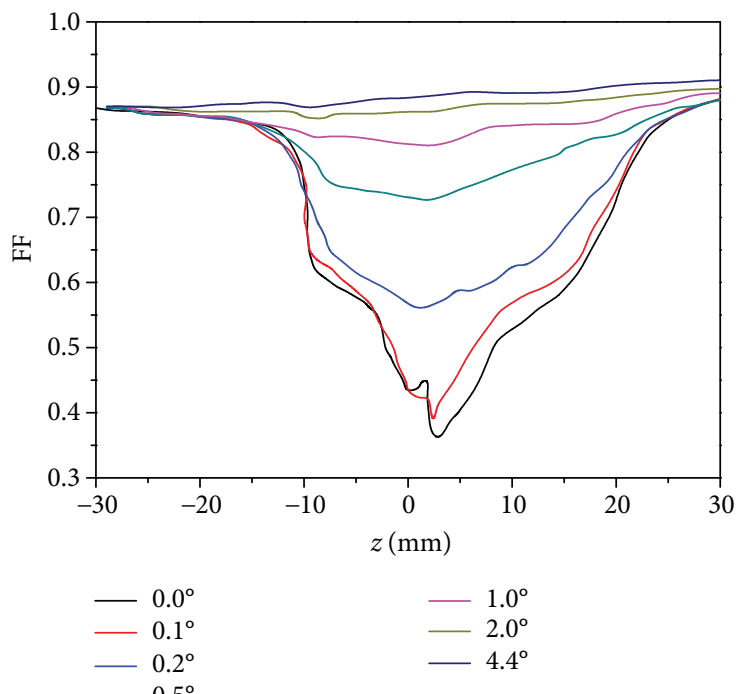

(c)

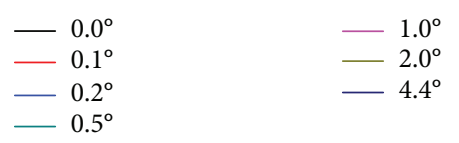

(b)

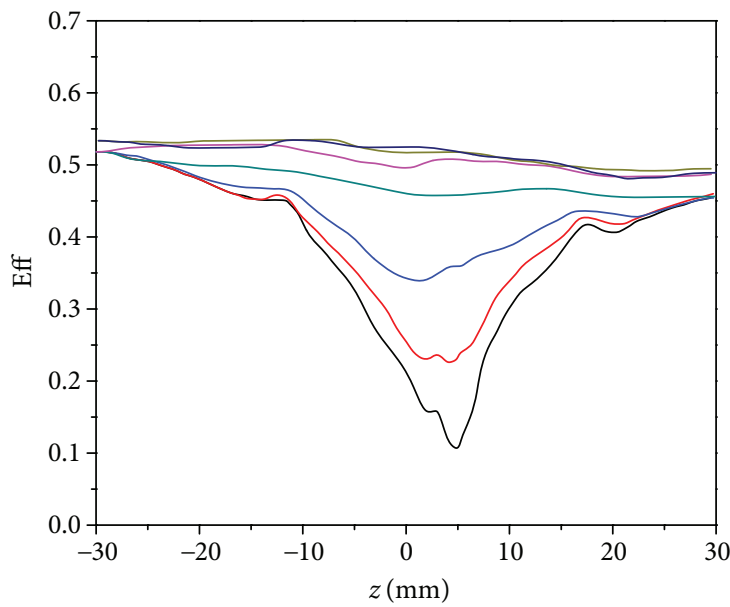

$0.0^{\circ}$
$0.1^{\circ}$
$0.2^{\circ}$
$0.5^{\circ}$

$-1.0^{\circ}$

$2.0^{\circ}$
$-\quad 4.4^{\circ}$

(d)

FIGURE 10: Variation of the cell characteristic parameters with the optical axis position under different divergence angles of light sources (cell size $5 \mathrm{~mm}^{*} 5 \mathrm{~mm}$ ): (a) short circuit current; (b) maximum-output power; (c) fill factor; (d) cell efficiency.
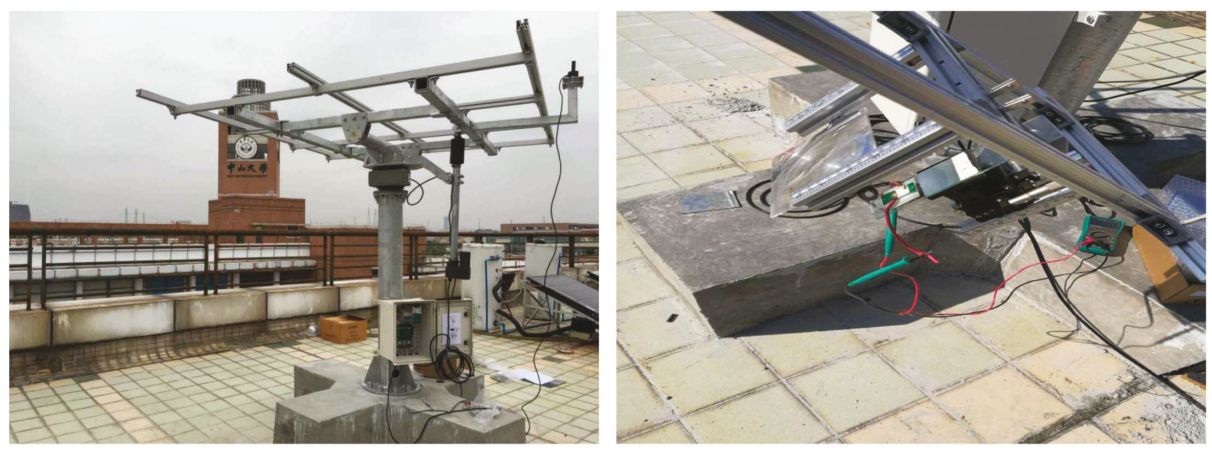

FIgURE 11: Experimental platform and device. 


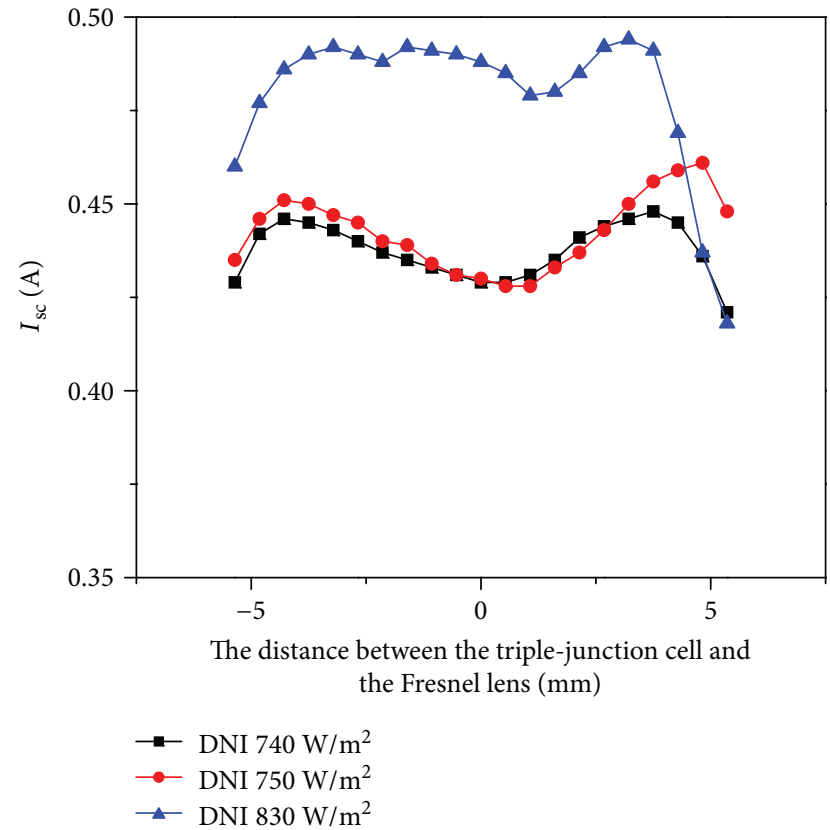

FIGURE 12: The short circuit current of the triple-junction cell along with the change of optical axis positions.

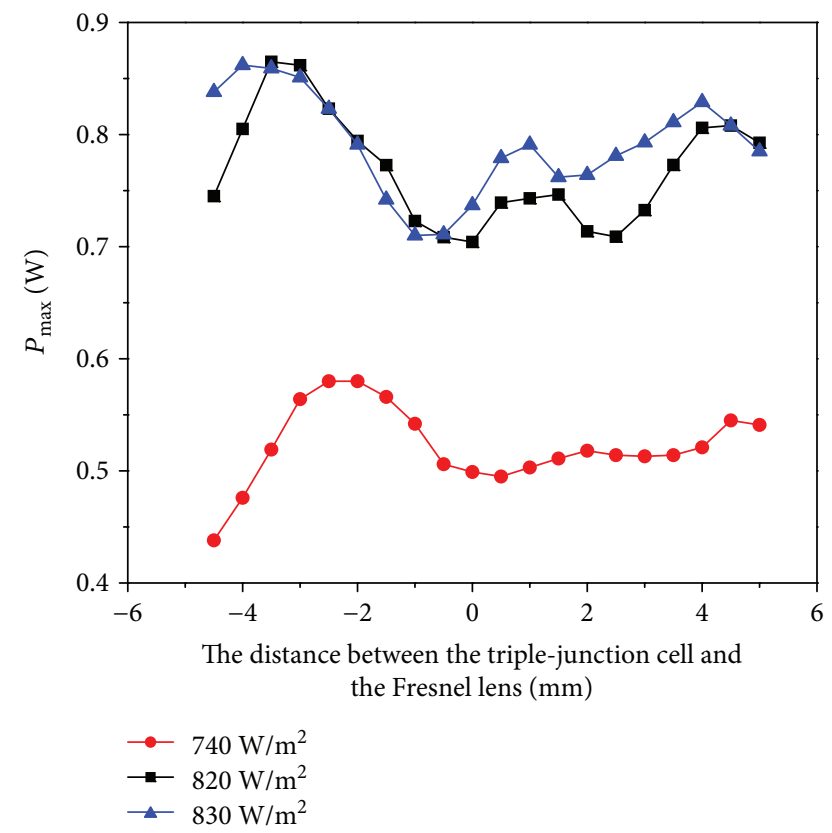

FIGURE 13: Changes in the maximum-output power of the triplejunction cell with the position of the optical axis.

due to the dispersion phenomenon of the light through the lens and the nonparallel incidence of light, the location of Fresnel's focus, the distribution of light, and the size of spot are different after the lens. Then, we simulated the photoelectric performance of the triple-junction cell. It is found that, at the focal plane of the lens, due to the difference of illumination distributions between three wave bands, there is current mismatch between the subcells of the triple-junction cell,

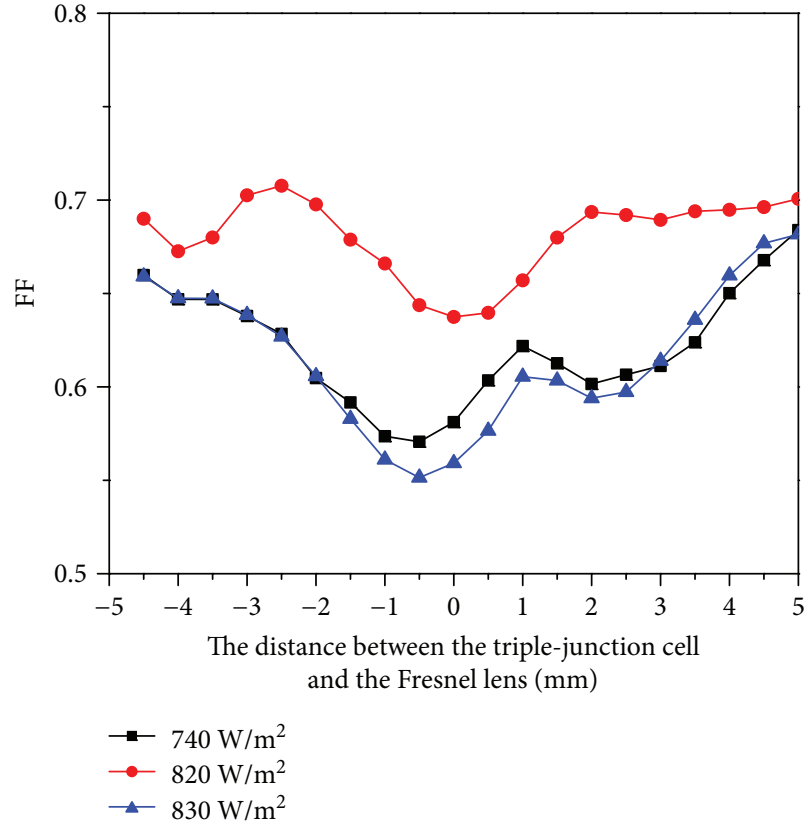

FIgURE 14: Changes in the fill factor of the triple-junction cell with the position of the optical axis.

causing the decrease of the short circuit current. And, due to the greater inhomogeneity of the illumination at the focal plane of the lens, the fill factor of the cell decreases. Therefore, there is a minimum value of the short circuit current, maximum-output power, and fill factor of the triplejunction cell in the focal position of the Fresnel lens, and there is a maximum value on both sides of the focal point. The output power on both sides of the focal point is increased by more than $15 \%$ over that of the focal plane. The simulation results are verified by the experimental results.

\section{Data Availability}

The data used to support the findings of this study are available from the corresponding author upon request.

\section{Conflicts of Interest}

The authors declare that they have no conflicts of interest.

\section{Acknowledgments}

The authors acknowledge the financial support of the National Natural Science Foundation of China (U1707603).

\section{References}

[1] H. Helmers, M. Schachtner, and A. W. Bett, "Influence of temperature and irradiance on triple-junction solar subcells," Solar Energy Materials and Solar Cells, vol. 116, pp. 144-152, 2013.

[2] W. Zhang, C. Chen, R. Jia et al., "Analysis of the interdigitated back contact solar cells: the n-type substrate lifetime and wafer thickness," Chinese Physics B, vol. 24, no. 10, article 108801, 2015. 
[3] E. F. Fernández and F. Almonacid, "A new procedure for estimating the cell temperature of a high concentrator photovoltaic grid connected system based on atmospheric parameters," Energy Conversion and Management, vol. 103, pp. 1031-1039, 2015.

[4] F.-X. Chen, L.-S. Wang, and W.-Y. Xu, "Enhanced optical absorption by Ag nanoparticles in a thin film Si solar cell," Chinese Physics B, vol. 22, no. 4, article 045202, 2013.

[5] G. Zubi, J. L. Bernal-Agustín, and G. V. Fracastoro, "High concentration photovoltaic systems applying III-V cells," Renewable and Sustainable Energy Reviews, vol. 13, no. 9, pp. 2645-2652, 2009.

[6] N. F. Chen and Y. M. Bai, "Concentrating photovoltaic system," Physics, vol. 36, p. 862, 2007.

[7] G. H. Yang, M. Wei, B. Z. Chen, M. C. Dai, L. M. Guo, and Z. Y. Wang, "Design and research of equal-thickness slab Fresnel lens," Journal Applied Optics, vol. 898, p. 34, 2013.

[8] F. Languy, K. Fleury, C. Lenaerts et al., "Flat Fresnel doublets made of PMMA and PC: combining low cost production and very high concentration ratio for CPV," Optics Express, vol. 19, no. S3, p. A280, 2011.

[9] M. A. Green, K. Emery, Y. Hishikawa, W. Warta, and E. D. Dunlop, "Solar cell efficiency tables (version 45)," Progress in Photovoltaics: Research and Applications, vol. 23, no. 1, pp. 1-9, 2015.

[10] M. Steiner, A. Bösch, A. Dilger et al., "FLATCON ${ }^{\circledR}$ CPV module with $36.7 \%$ efficiency equipped with four-junction solar cells," Progress in Photovoltaics: Research and Applications, vol. 23, no. 10, pp. 1323-1329, 2015.

[11] Y. Ota and K. Nishioka, "Three-dimensional simulating of concentrator photovoltaic modules using ray trace and equivalent circuit simulators," Solar Energy, vol. 86, no. 1, pp. 476-481, 2012.

[12] M. Steiner, S. P. Philipps, M. Hermle, A. W. Bett, and F. Dimroth, "Validated front contact grid simulation for GaAs solar cells under concentrated sunlight," Progress in Photovoltaics: Research and Applications, vol. 19, no. 1, pp. 73-83, 2011.

[13] M. Steiner, W. Guter, G. Peharz, S. P. Philipps, F. Dimroth, and A. W. Bett, "A validated SPICE network simulation study on improving tunnel diodes by introducing lateral conduction layers," Progress in Photovoltaics: Research and Applications, vol. 20, no. 3, pp. 274-283, 2012.

[14] G. Segev, G. Mittelman, and A. Kribus, "Equivalent circuit models for triple-junction concentrator solar cells," Solar Energy Materials and Solar Cells, vol. 98, pp. 57-65, 2012.

[15] P. Rodrigo, E. F. Fernández, F. Almonacid, and P. J. PérezHigueras, "Models for the electrical characterization of high concentration photovoltaic cells and modules: a review," Renewable and Sustainable Energy Reviews, vol. 26, pp. 752760, 2013.

[16] S.-G. Yi, W.-H. Zhang, B. Ai, J.-W. Song, and H. Shen, “Analysis of each branch current of serial solar cells by using an equivalent circuit model," Chinese Physics B, vol. 23, no. 2, p. $028801,2014$.

[17] X. J. Jia, B. Ai, X. X. Xu, J. M. Yang, Y. J. Deng, and H. Shen, "Two-dimensional device simulation and performance optimization of crystalline silicon selective-emitter solar cell," Acta Physica Sinica, vol. 63, article 068801, 2014.
[18] Q. B. Liang, B. F. Shu, L. J. Sun, Q. Z. Zhang, and M. B. Chen, "Optimization of light spot intensity and coverage to a triplejunction solar cell under non-uniform illumination," Acta Physica Sinica, vol. 63, article 168801, 2014.

[19] Q. B. Liang, "The optimization on electrical performance and thermal stress of high-concentration CPV modules," $\mathrm{Ph}$. D. Dissertation (Sun Yat-sen University), Guangzhou, China, 2015. 

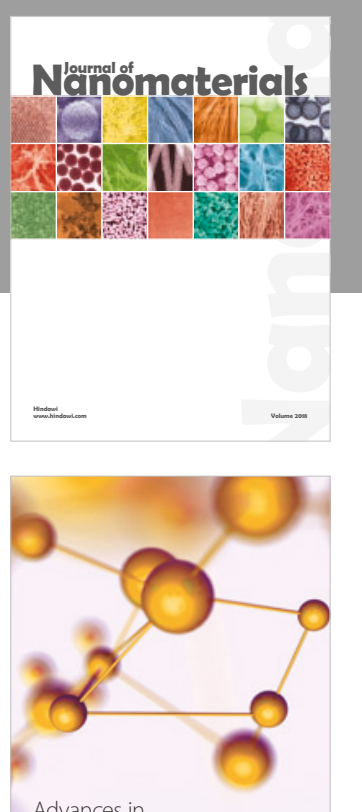

Physical Chemistry
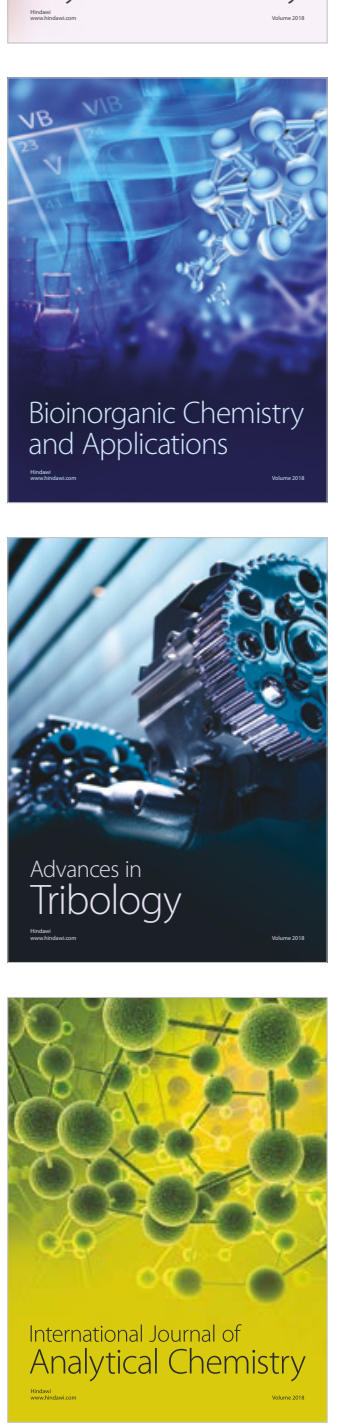

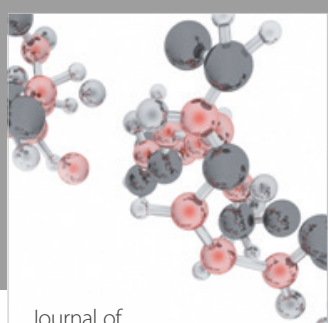

Analytical Methods

in Chemistry

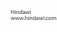

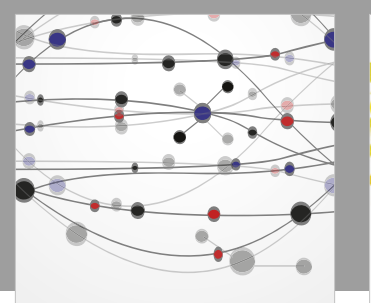

The Scientific World Journal

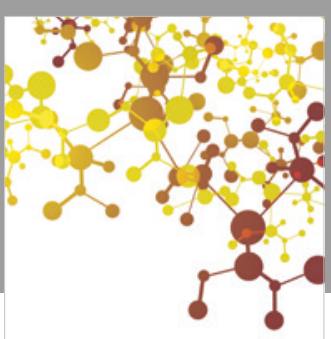

Journal of

Applied Chemistry
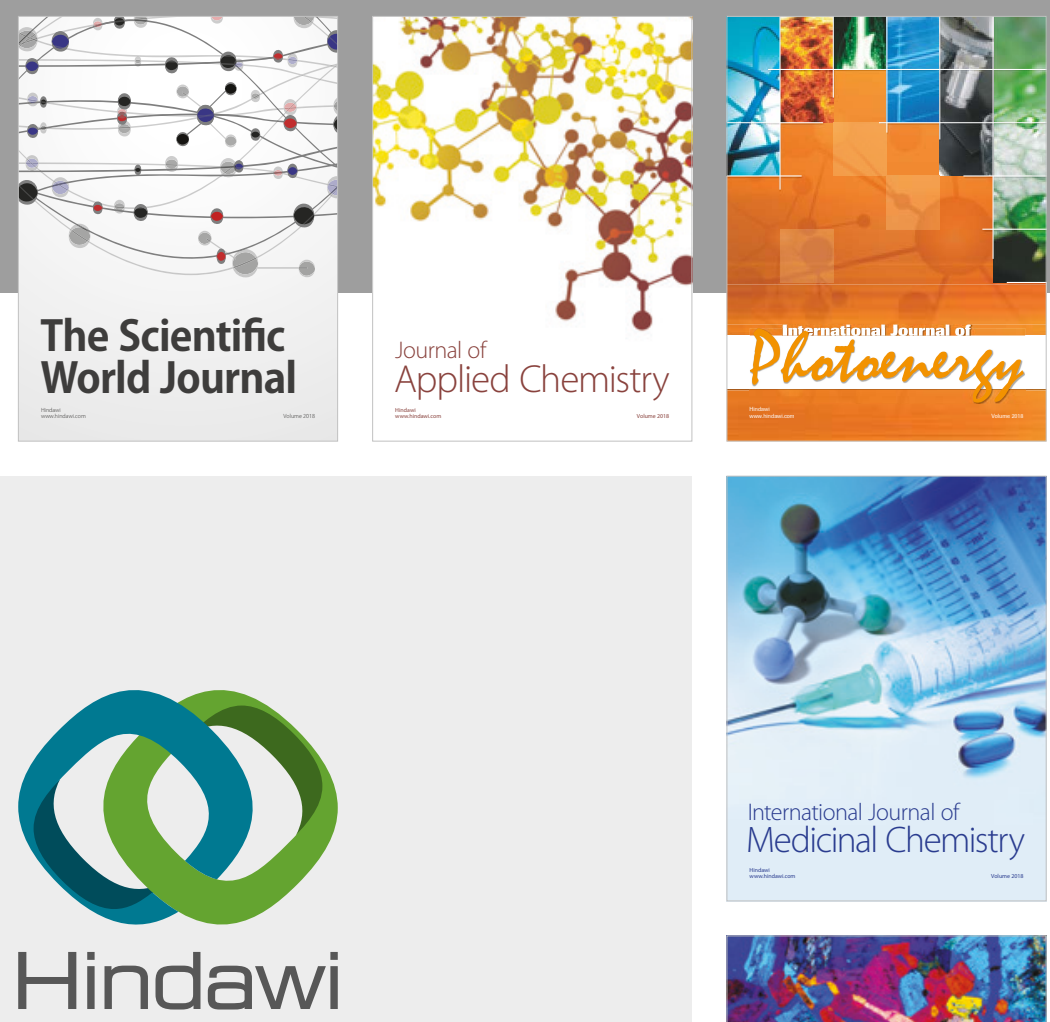

Submit your manuscripts at

www.hindawi.com
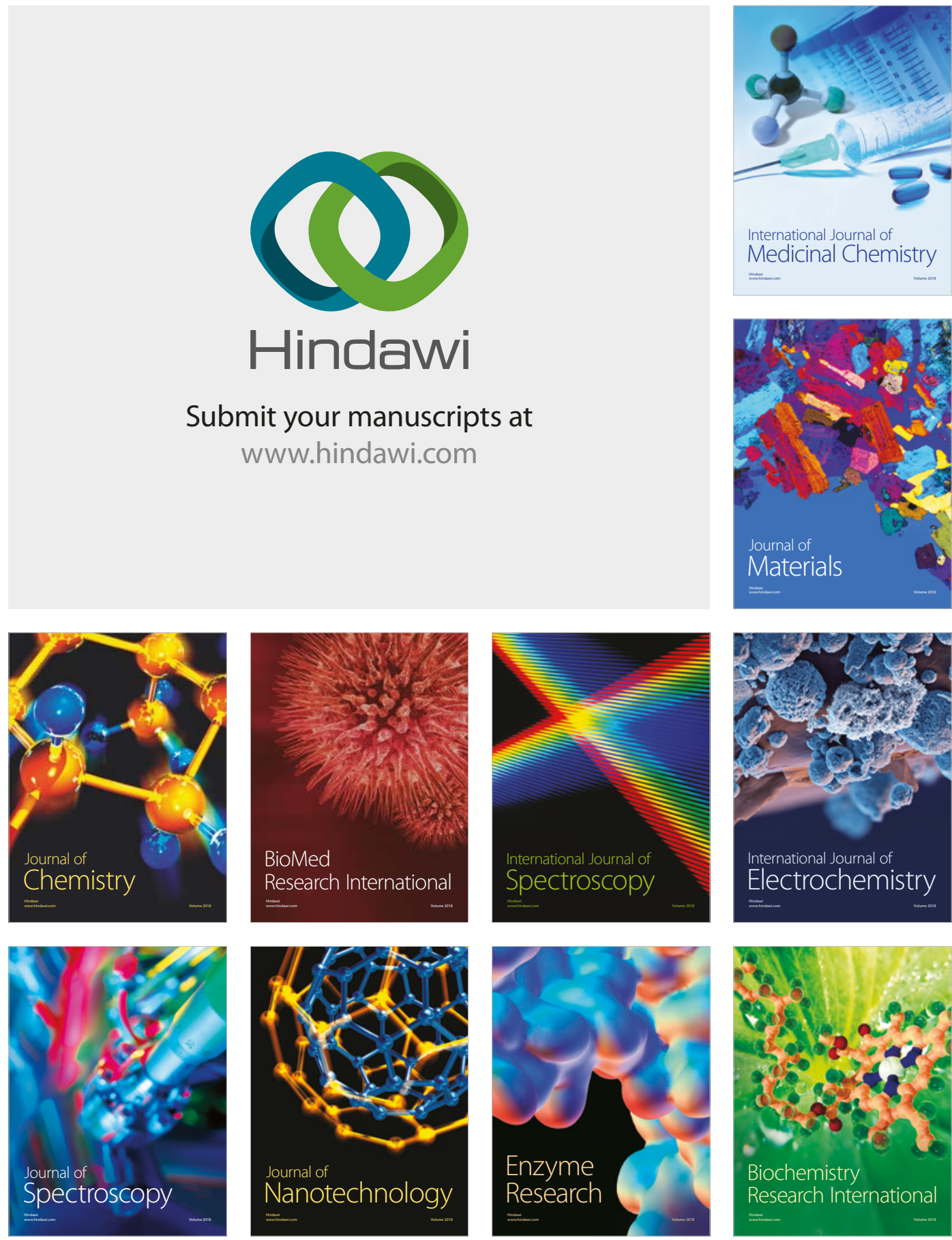
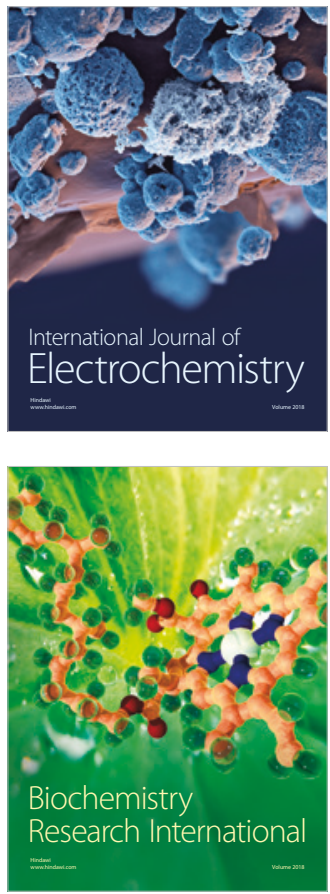